\title{
Transforming a competency model to parameterised questions in assessment
}

\author{
Onjira Sitthisak, Lester Gilbert, and Hugh C Davis \\ Learning Societies Lab, School of Electronics and Computer Science, University of \\ Southampton, Highfield, Southampton, SO17 1BJ, United Kingdom \\ \{os05r, lg3, hcd \}@ecs.soton.ac.uk
}

\begin{abstract}
The problem of comparing and matching different learners' knowledge arises when assessment systems use a one-dimensional numerical value to represent "knowledge level". Such assessment systems may measure inconsistently because they estimate this level differently and inadequately. The multi-dimensional competency model called COMpetence-Based learner knowledge for personalized Assessment (COMBA) is being developed to represent a learner's knowledge in a multi-dimensional vector space. The heart of this model is to treat knowledge, not as possession, but as a contextualized space of capability either actual or potential. The paper discusses a system for automatically generating questions from the COMBA competency model as a "guide-on-the-side". The system's novel design and implementation involves an ontological database that represents the intended learning outcome to be assessed across a number of dimensions, including level of cognitive ability and subject matter. The system generates all the questions that are possible from a given learning outcome, which may then be used to test for understanding, and so could determine the degree to which learners actually acquire the desired knowledge.
\end{abstract}

Keywords: competency, assessment, knowledge level, ontology

\section{Introduction}

In recent years, a variety of tools and learning environments have been created and installed in schools, universities, and organisations to support learning. Mostly these tools have been created to support e-learning content and collaborative learning activities like a virtual classroom [1]. However, e-learning suggests not only new technologies for instruction but also new pedagogical approaches to enhance learning. One new pedagogical approach is machine-processable competency modelling. A competence model is introduced for storing, organizing and sharing learners' performance data in order to seek and interpret evidence for where the learners are in their learning, where they want to go, and how they can get there. Pedagogically effective and informed competency data is vital in any assessment system.

One of the desired outcomes of an assessment system is information about the learners' knowledge, identifying what learners can do by representing their current 
state of knowledge [2]. This information is collected and updated during the assessment process. Most assessment systems assume that knowledge is something that a learner possesses or fails to possess, and seek to estimate a learner's "knowledge level". As a result, such assessment systems may measure "knowledge level" inconsistently because they estimate this level differently, and inadequately because they use one-dimensional numerical values [3]. The proposed solution is to consider the learners' "learned capability" instead of their "knowledge level", and to consider competencies and learned capabilities as a multidimensional space.

In the context of an adaptive assessment system, an assessment is part of the process of diagnosing the learner's competence. The key idea of an adaptive assessment system is that questions are selected by the computer to individually match the learner's competence [4]. The system's evaluation of the learner's competence is then used to guide the adaptation of the system [5]. The system may skip over what learners have learned and find out what they should learn further. While an adaptive system may be more efficient for summative assessment, a system of adaptive formative assessment is likely to be of greater advantage to learners, since they would receive relevant, personalized feedback. Establishing adaptive formative assessment systems to support lifelong learning is extremely challenging and relies on introducing a competency model to the adaptive assessment. Our intention is not to promote a particular technological platform, but to demonstrate how a competency model can be applied to adaptive assessment.

In this paper, we introduce an advanced competency model named COMpetenceBased learner knowledge for personalized Assessment (COMBA). The COMBA model is represented in a multi-dimensional vector space. We explore the assembly of competencies into a tree structure and then consider the task of adaptively generating assessments from such a competencies structure. Finally, an implementation of COMBA is presented.

\section{The Multi-dimensional COMBA model}

Competence-based approaches in the field of e-learning, institutional admissions, learners seeking courses, e-portfolios, job references, human resource management, and job descriptions are becoming more common. They appear to offer the opportunity to develop tools and services for data exchange, discovery, processing, analysis, and visualization to meet needs of learners, tutors, program managers, examination bodies, professional societies, employers, legislators, and so on. We suggest that a complete and coherent model of competencies would support storing, organizing and sharing of achieved, current, and intended performance data relating to all aspects of education and training in a persistent and standard way [3]. We have been developing a competency model, named COMBA, which is proposed for all domains where learning and teaching take place.

In the first stage of developing the model [3], we conceptualised "competency" as involving a capability associated with a given subject matter content, requiring a proficiency level, and associated with evidence, any required tools, and a definition of the situation which contextualizes the competency. In the second stage of developing 
the model [6], we implemented an exemplar UK Royal College of Nursing competency [7] reflecting relevant features of a learner's behaviour and knowledge that affected their learning and performance. An outcome of this implementation exposed a critical issue involving the expression of ethical practice in the COMBA model. One of the conceptions of competence for a nursing graduate is competence in ethical practice [8] as well as the other characteristics of professional service delivery involving knowledge and psychomotor skill [9]. Hence, attitude, the way in which a learner exhibits their knowledge and skill, is included in the COMBA model, as illustrated in Fig. 1.

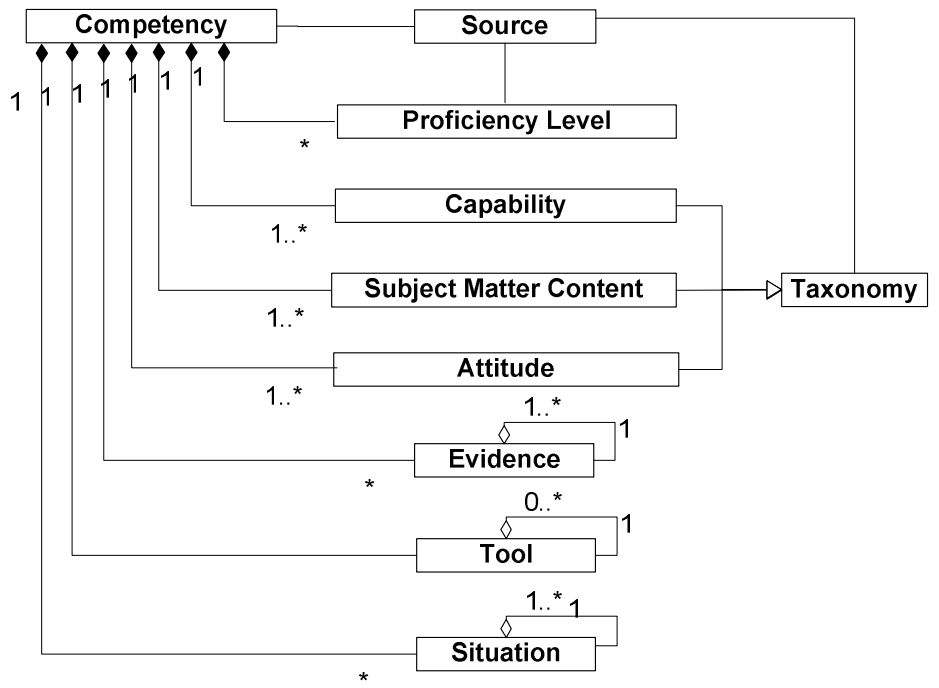

Fig. 1. Competency model including attitude component

The COMBA model considers knowledge in the widest possible sense, and involves the following four major components: subject matter, capability, attitude, and context, along with metadata as illustrated in Fig. 2.

The challenge of capturing and using knowledge starts with the problem of understanding its nature and representation. The failure of previous efforts to 'intelligently process knowledge' (e.g. intelligent tutoring systems) may be due to their pedagogically and cognitively inadequate characterization of this knowledge, and their simplistic assumptions that knowledge is something a learner possesses or fails to possess.

The heart of the COMBA model is to treat knowledge, not as possession, but as a contextualized multidimensional space of capability either actual or potential. Accordingly, the three important components of the COMBA model (capability, subject matter content, and attitude), which are referred from relevant taxonomies or ontologies, may be represented in a vector space as in Fig. 3. The learned capability is the learner's required or observed behaviour, for example using Bloom's taxonomy [10]. The subject matter content in Fig. 3 is based on Merrill's analysis [11], and attitude is based on a version of Krathwohl's taxonomy [12]. 


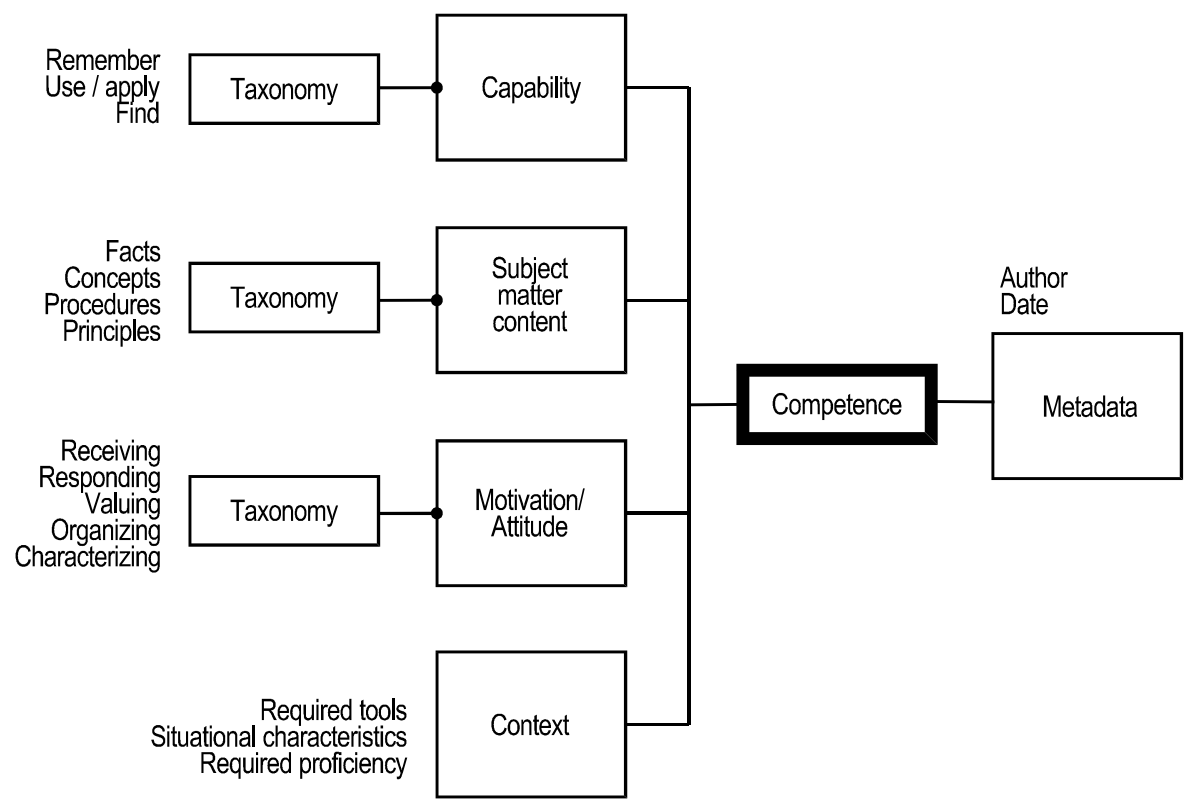

Fig. 2. Individual competence model

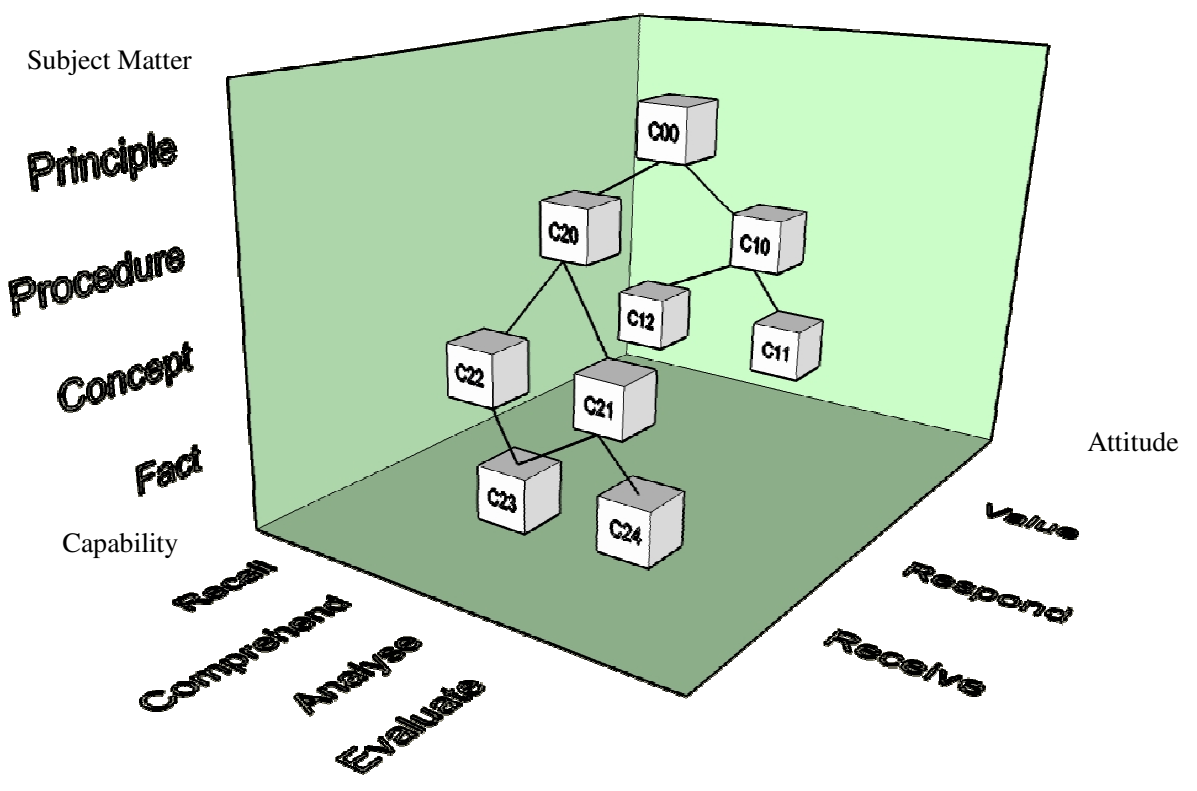

Fig. 3. Multidimensional space of competency model 
In this paper, we choose competencies from health care because they are amongst the most sophisticated and challenging to implement [13]. Table 1 represents some nursing competencies based on the multidimensional space of the COMBA model. For example, $\mathrm{C} 00$ (students are able to use and value ethical principles) comprises C10 (students are able to actively apply ethical principles) and C20 (students are able to actively use professional regulation). In order to achieve $\mathrm{C} 10$, students should be able to demonstrate client confidentiality respectfully (C11), and to identify ethical issues sensitively (C12). In order to achieve C20, students should be able to identify the limitations in their own practice $(\mathrm{C} 22)$, and to considerately evaluate professional regulation $(\mathrm{C} 21)$. There is a common competency for $\mathrm{C} 21$ and $\mathrm{C} 22$ which is $\mathrm{C} 23$ (students are able to recognize the need for referral willingly). In order to achieve $\mathrm{C} 21$, students should be able to recall relevant professional regulations willingly (C24). This shows that we can map effectively these more complicated competencies into the COMBA model. The subject matter, capability taxonomy, attitude taxonomy, and competence were ontologically represented based on the Simple Knowledge Organisation System (SKOS) [14].

Table 1. Some example nursing competencies represented in the competency model

\begin{tabular}{clll}
\hline Competency No. & Capability & Subject Matter Content & Attitude \\
\hline C00 & Use & Ethical principles & Values \\
C10 & Apply & Ethical issues & Actively \\
C11 & Demonstrate & Client confidentiality & Respectfully \\
C12 & Identify & Ethical Issues & Sensitively \\
C20 & Use & Professional regulation & Actively \\
C21 & Evaluate & Professional regulation & Considerately \\
C22 & Identify & Limitation in own practice & Values \\
C23 & Recognize & Need for referral & Willingly \\
C24 & Recall & Professional regulations & Willingly \\
\hline
\end{tabular}

\section{The Competency tree}

Competencies are assembled into trees. A tree structure is a particular way of representing a structure in a graphical form [15]. While the relationship between nodes is modelled as a family relation such as parent and child, there is no ordering of nodes on the same level, and this yields a tree structure rather than a hierarchy. It is assumed that all children of a defined competency are required in order to achieve proficiency for the parent. While the tree structure defines a top-down or bottom-up structure, it does not imply sequencing as might be implied in a hierarchy. For example, a competency tree may specify how to roll up the assessment for each competency throughout a competency tree without implying sequencing of assessments of same level competencies. So the issues of pedagogical sequencings are not considered at this stage by representing competencies as a tree structure instead of a hierarchy.

One of the advantages of a competence tree structure is that a tree structure separates the composition rule in the domain from other structural components. 
Hence, an application of the competency model, such as in adaptive assessment, may add other rules, perhaps based on pedagogical sequencing, in order to control the adaptation within the competency tree.

More technically, the COMBA model specifies the network of assembled competencies as a directed acyclic graph. In competency terms, Fig. 4 implies that competency $C 00$ is decomposed into sub-competencies $C 10$ and $C 20$, such that $C 10$ and $C 20$ contribute to $C 00$. A node may have more than one parent, provided the parent is not a child of the node. Fig. 4 shows a "forest" of two competency trees, where arrows represent parent-child relationships. A competency tree may specify common children for more than one node, or more than one origin node. For example, $\mathrm{COO}$ and $\mathrm{A}$ represent different competencies that have certain competencies in common such as $C 22$.

It is expected that competency trees will be different for different communities and users. For example, a tree of nursing competencies from the UK Royal College of Nursing would have many points of difference from a similar tree from the Canadian Nursing Association. At a personal level, a student nurse may develop his or her own tree to reflect their own competencies, both achieved and to be attained.

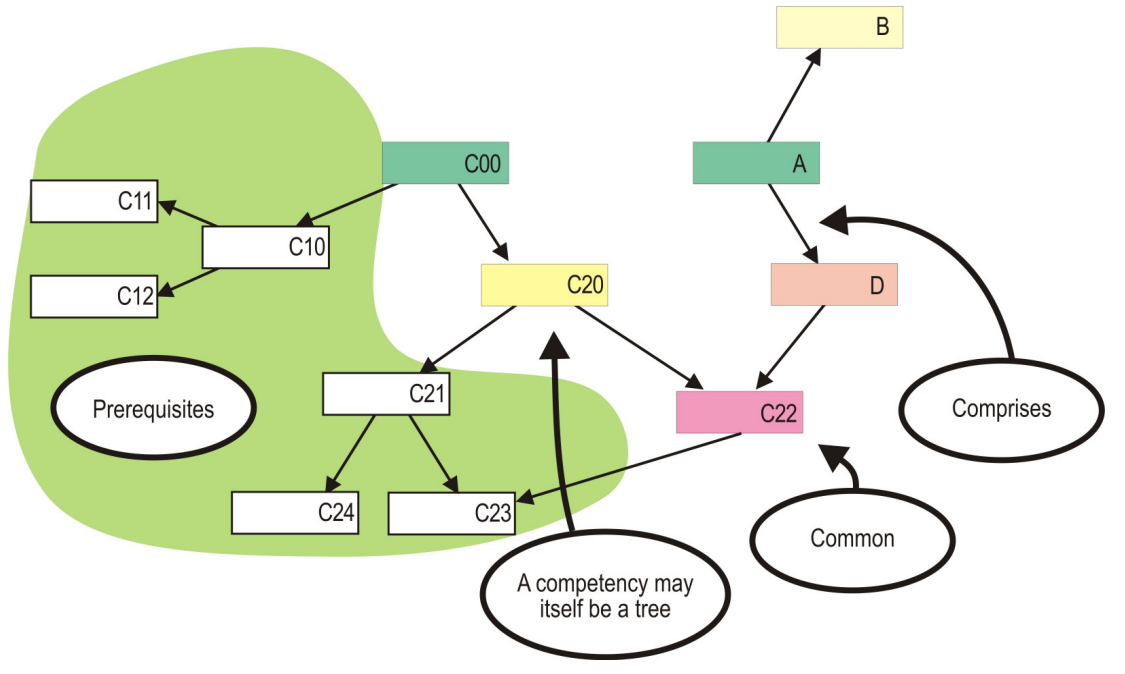

Fig. 4. Competency tree

\section{Generating assessment items from a competency tree}

Assessments may be categorized as formative, summative, or diagnostic [16]. Formative assessment provides prescriptive feedback to assist learners in reaching their competences [17]. It is intended to help the learner deal with deficiencies in their understanding, knowledge, or competence. In contrast, summative assessment is generally given at the end of a period of learning to establish what knowledge, skills, and/or attitudes the learner has acquired over a period of time. It helps to establish whether learners have attained the competences required, and is not focussed on 
supporting learning. Diagnostic assessment is an in-depth assessment related to strengths and weaknesses in each skill area, which identifies priorities and needs [18]. It helps to determine what learners can already do within the goals of the curriculum. This paper focuses on formative and diagnostic assessment.

There are two problems of traditional formative assessment. First, learners are likely to need different kinds of formative assessment at different stages in their 'learning journeys' [19]. Second, formative assessment usually only provides a list of the learner's deficiencies [17] without clearly specifying their boundaries. These problems are relieved by using an assessment tree suggested in this paper.

\subsection{Constructing an assessment item}

We assume an assessment which takes place in the context of the COMBA model. The competency tree might be used to drill down into component competencies for the tested competency, helping to define what to test and how to test it. An assessment for a competency often actually tests component competencies. For example, a paediatric nurse course [20] may test knowledge of professional regulation by testing the learners' ability to demonstrate and evaluate understanding of professional regulation including the demonstration of a variety of specific skills and attitudes, as illustrated in Table 1.

A generic assessment item can be directly formulated from a competence specification by using the parameters of that competence: capability, subject matter content, attitude and other contexts such as tool and situation as the authoring question templates in Table 2. For example, the assessment corresponding to C11 might be something like "What information must be kept confidential in situation A?", or "Identify the information which doesn't need to be kept confidential in situation B", as illustrated in Table3.

Table 2 Question templates

\begin{tabular}{cl}
\hline No. & \multicolumn{1}{c}{ Question Templates } \\
\hline $\mathrm{a}$ & {$[$ Capability $]+[$ Subject $]$} \\
$\mathrm{b}$ & {$[$ Capability $]+[$ Subject $]+[$ Situation $]$} \\
$\mathrm{c}$ & {$[$ Capability $]+[$ Subject $]+[$ Attitude $]$} \\
$\mathrm{d}$ & {$[$ Subject $]+[$ Situation $]$} \\
\hline
\end{tabular}

Table 3 Some example questions represented from the competencies

\begin{tabular}{cclc}
$\begin{array}{c}\text { Competency } \\
\text { No. }\end{array}$ & $\begin{array}{c}\text { Question } \\
\text { No. }\end{array}$ & \multicolumn{1}{c}{ Question } & $\begin{array}{c}\text { Template } \\
\text { No }\end{array}$ \\
\hline C00 & Q1 & $\begin{array}{l}\text { Identify the outcomes if ethical principles were not } \\
\text { valued. }\end{array}$ & $\mathrm{c}$ \\
& Q2 & $\begin{array}{l}\text { List ethical principles. } \\
\text { What ethical principles are involved [in situation X]? }\end{array}$ & $\mathrm{a}$ \\
\hline Q3 & Q4 & $\begin{array}{l}\text { Identify the possible outcomes if ethical issues were } \\
\text { not actively applied. }\end{array}$ & $\mathrm{c}$ \\
& Q5 & $\begin{array}{l}\text { How would you apply ethical issues [in situation Y]? } \\
\text { Define the specific ethical issues [in situation Z]. }\end{array}$ & $\mathrm{b}$ \\
& Q6 & b & \\
\hline
\end{tabular}


A formative assessment may contain items to test finer grained competencies. A competency tree can be used as a guide to assemble the necessary set of test items for assessing each competency. In this process, the competency tree is transformed to an assessment tree. An assessment tree consists of question nodes from Table 3, where each question node corresponds to a competency node, as illustrated in Fig. 5.

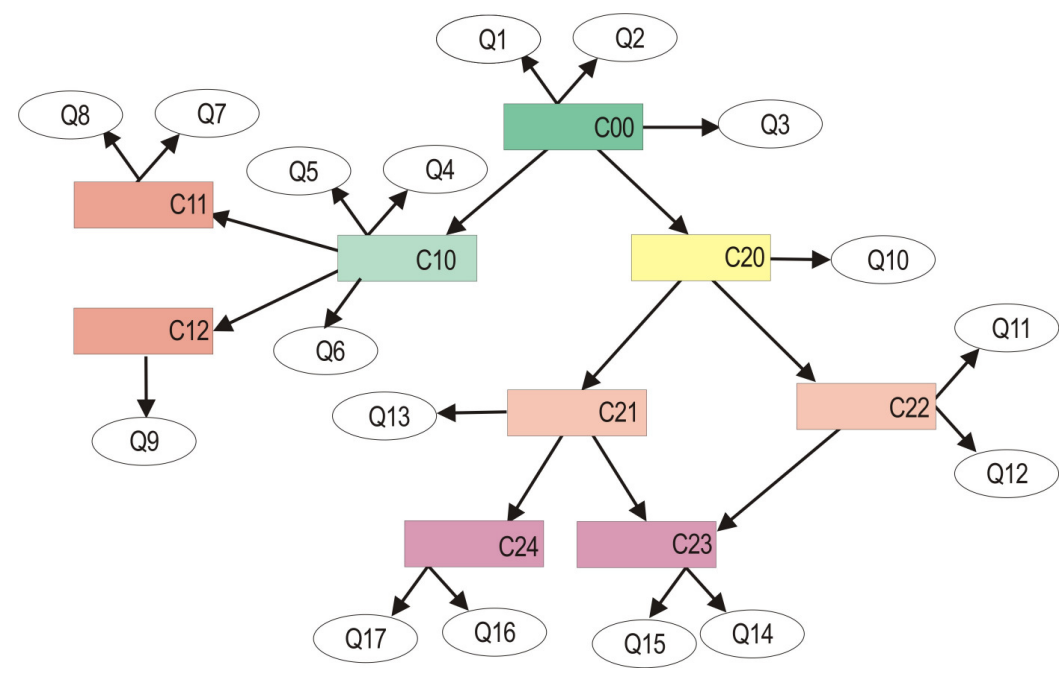

Fig. 5 The group of questions based on a competency tree

Different organizations or communities of practice may have different processes and policies for assessment. By specifying a particular competency tree or sub-tree to be assessed, it is possible to align the assessment needed based on the needs of the organization or community of practice. Hence, the competency tree defines a standard way to specify explicitly the component competencies to be assessed, and provides a "guide on the side", automatically generating a set of general assessment items.

\subsection{Navigating assessment items on the competency tree}

There are a number of adaptive assessment methods and technologies that can be used to assess learners' strengths and weaknesses based on item-by-item and learner responses. These allow learners to be tested on materials at a level appropriate to their current understanding. Adaptive assessments change their behaviour and structure depending on the learners' responses and inferred abilities.

There are two major adaptation techniques; presentational adaptation and navigational adaptation [21]. An adaptive system may apply these two techniques with questions. Traversing the competency tree may start at the leaf node or the root node depend on the objective of each application. As a result, a competency tree may be traversed, mapped, extended, visualized, and searched by a variety of applications and tools. For example, a competency tree may be used to specify how to roll up the 
assessments for each competency in order to personalize the assessment and match assessment items to the individual competences of each learner.

There is a set of possible assessment items associated with each competence node, as illustrated in Fig. 5. Given a pruned competency tree (a tree whose remaining branches and leaves represent competencies not yet attained by a learner), an adaptive assessment system now needs to sequence the assessment items. Sequencing could be based upon pedagogical considerations, and arranged according to the taxonomies of subject matter content [11], of capability [10], and so on. For example, an adaptive assessment system may start with assessment items at the lower level of the capability taxonomy and progress to the higher levels, in order to reach the boundary of the learner's understanding. On the other hand, sequencing could be based on the learner's preferences. Depending on the learner's answers, the next assessment item will be presented. This involves regenerating the sequence based on the learner's unfolding competences. The result of an adaptive assessment partitions the competency tree into "what the student can do" and "what the student is ready to learn" [22] and finding the boundaries of competence for the learner.

\section{Implementation}

COMBA aims to provide a system which is able to accommodate complicated competencies, link competencies adequately, and support tracking of the knowledge state of the learner. This makes identification of the assessment that would demonstrate successful teaching and learning straightforward. The system focuses on the identification and integration of appropriate subject matter content (represented by a hierarchy of competencies) and cognitive ability (represented by a capability taxonomy). A simplified COMBA system was implemented in this study by omitting the dimension of "attitude".

The system was built upon an ontological database that describes all resources and the relationships between them. The advantage of ontological schemas over database schemas is that ontological schemas define explicit formal specifications and include machine-interpretable definition to share common understanding of the structure of information among people or software agents [23]. Thus, the ontological database is flexible and extensible, allowing the resources in the system to be described on the Semantic Web, interoperability between different systems, and reasoning about the described resources.

COMBA consists of a number of modules (illustrated in Fig. 6): competence navigator, subject matter navigator, capability navigator, question assembler, question to QTI schema converter and sequencing manipulator. The competence navigator is responsible for retrieving the requested competence based on the domain request from the user, and passing the competence to the subject matter and capability navigator modules. The relevant subject matter and capability data received from those modules, together with the authoring question template files, are assembled to generate questions derived from the matrix of competencies crossed with cognitive abilities. Then, the questions are formatted according to the IMS Question and Test Interoperability specification (IMS QTI) standard [24]., enabling the sharing of the 
questions and tests. In order to develop a test, the generated questions are linked together for storing in a test bank. For the delivery of the assessment, the system deploys an assessment delivery service (ASDEL) (http://www.asdel.ecs.soton.ac.uk/) to allow a learner to view a question and answer it. In the next stage of the research, the system will be extended to marking and feedback.

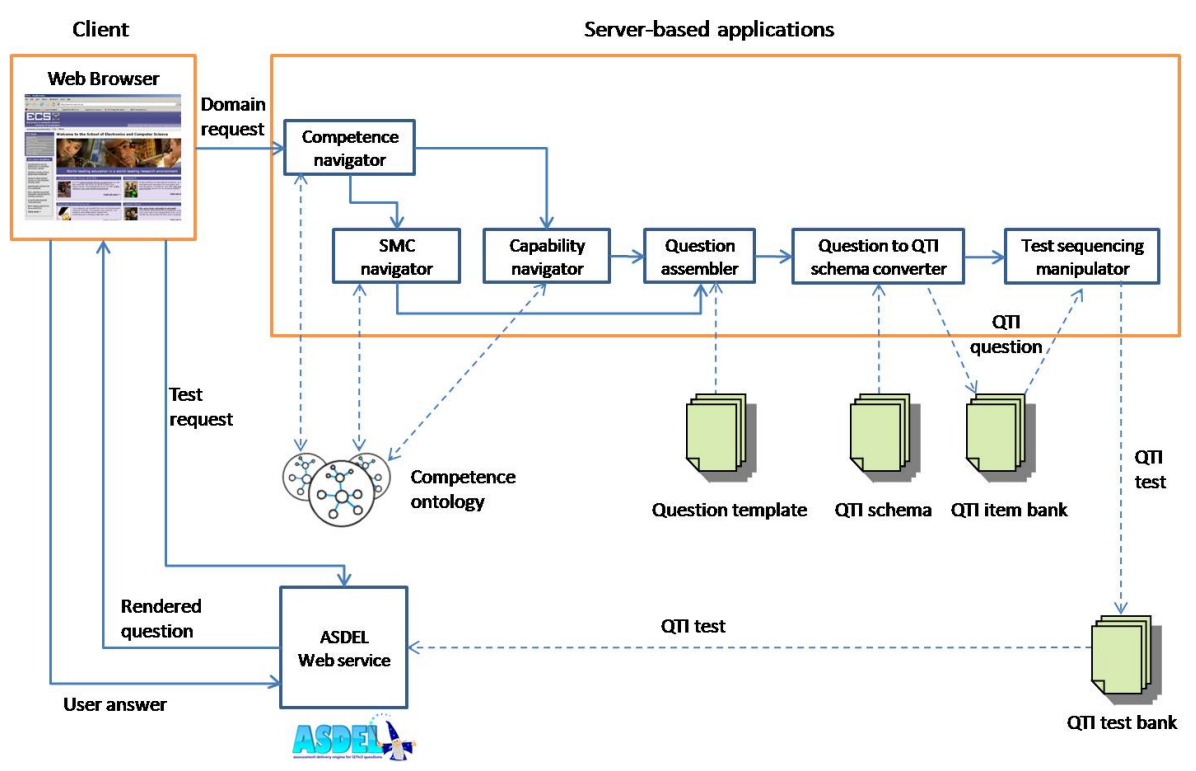

Fig. 6 Architecture for the COMBA system

The following section presents data creation, representation and storage, methods of generating and standardizing questions, and methods of question delivery in the COMBA system.

\subsection{Data creation, representation and storage}

A domain expert expressed domain content, the capability taxonomy, and competence in an English-like form. A knowledge engineer represented these elements in the form of a semantic network, and then transformed them into an ontology. The ontologies adhered to the criteria of ontology design: clarity, coherence, extendability, minimal encoding bias, and minimal ontological commitment [25]. These ontologies are domain, not structure, ontologies using a controlled vocabulary from SKOS [26]. Sharing and reuse of information are integral aspects of the Semantic Web. In the COMBA system, the ontology was based on Semantic Web technology standards of RDF [27] and OWL [28]. The ontology of the COMBA system is shown in Fig. 6. The definitions of the elements in the competence ontology are shown in Table 4. 


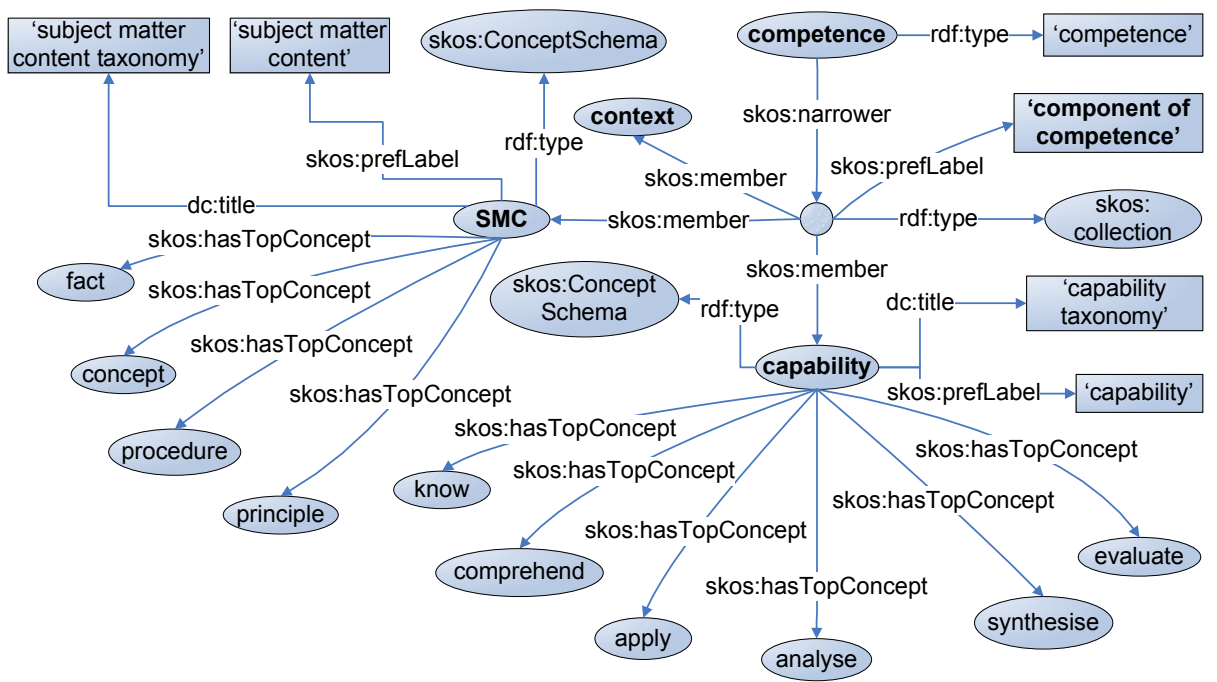

Fig. 7 Ontology of COMBA

Table 4 the definitions of each element in the competence ontology.

\begin{tabular}{|c|c|}
\hline Class & Definition \\
\hline Competence & $\begin{array}{l}\text { Defines a capability associated with subject matter content, a proficiency } \\
\text { level, evidence, any required tools, and definition of the situation which } \\
\text { contextualises the competency. }\end{array}$ \\
\hline SMC & $\begin{array}{l}\text { Defines the subject domain of what the learner can do by the end of the } \\
\text { unit of teaching and learning. }\end{array}$ \\
\hline Capability & $\begin{array}{l}\text { Defines behaviour that can be observed, based on a taxonomy of learning } \\
\text { such as Bloom's, Gagné's nine areas of skill, or Merrill's cognitive } \\
\text { domain. }\end{array}$ \\
\hline Context & $\begin{array}{l}\text { Defines the particular context and conditions of the competency, such as } \\
\text { tools and situations. }\end{array}$ \\
\hline Fact & $\begin{array}{l}\text { Defines statements, or factual information which consists of an attribute } \\
\text { and a value. }\end{array}$ \\
\hline Concept & $\begin{array}{l}\text { Defines a group of objects or ideas which are designated by a single word } \\
\text { or term. Area concept has a number of attributes which are used to classify } \\
\text { or categorise objects according to their values on those attributes. }\end{array}$ \\
\hline Procedure & Defines a sequential set of steps to accomplish a task or make a decision. \\
\hline Principle & $\begin{array}{l}\text { Defines cause-effect relationships describing the behaviour of a system. It } \\
\text { can usually be expressed as some sort of an equation if the system is in the } \\
\text { scientific or engineering domain. }\end{array}$ \\
\hline Know & \multirow{6}{*}{ Cognitive domain capabilities according to Bloom } \\
\hline Comprehend & \\
\hline Apply & \\
\hline Analyse & \\
\hline Synthesise & \\
\hline Evaluate & \\
\hline
\end{tabular}




\subsection{Method of generating questions}

In the system, when learners submit their domain of interest to the system, the competence navigator module navigates the competency using the competency ontological database based on the request, where relevant subject matter and capability nodes are retrieved. In this stage, both breadth-first and depth-first strategies can be implemented. The authoring question templates as shown in Table 5 are provided from a test instructor. The retrieved subject matter and capability nodes and the templates are assembled in questions. The process of traversing competencies, retrieving the relevant nodes and converting to questions are recursive. The generated questions are standardised for conformance to the QTI specification by a conversion process using the QTI schema. Finally, the QTI questions are sequenced using a desired strategy such as breadth-first or depth-first. The resulting test file is a sequenced set of questions.

\subsection{Method of question delivery}

In this research, ASDEL was deployed as a stand-alone web application in order to deliver the tests to the learners. The test files in the QTI test bank were queried from the ASDEL web service. ASDEL is responsible for allowing a learner to view a question and to answer it. The type of a question currently in the system is the essay type, so a teacher has to provide written criticism rather than relying on ASDEL to provide feedback. A Web service API including marking, giving feedback, and retrieving assessment results, will be extended in the next stage of the research.

\section{Conclusion}

We have proposed the next generation of a competency model named COMBA to support adaptive assessment. The COMBA model includes "attitude", identified as a critical issue exposed by working with nursing competencies, as well as including subject matter domain knowledge, and learned capabilities. The multi-dimensional COMBA model represents competency in terms of a tree structure.

The benefits of a COMBA-enabled adaptive system are to help learners identify and diagnose their boundaries of their own competencies, understand them, and find out how to progress by comparing them with a given or ideal competency tree. Adaptive assessment involves the dynamic sequencing of assessment items derived from the COMBA competency tree depending on the learner's responses.

Although the system can automatically generate a list of all the questions that are possible at various levels from a competency framework, we face the immediate challenge of representing the subject matter content based on the concept of a hierarchy of knowledge. The hierarchy of knowledge may be classified as fact, concept, procedure, and principle based on Merrill [11]. This needs to use a specialist or subject matter content expert to analyse the domain before a knowledge engineer can process it later, and may be regarded as a problem for the current system. 
A major challenge in the construction of a competency ontology is that the existing competencies in the course syllabus are required to be well-defined. This is usually not the case in most existing syllabi.

Acknowledgments. This work was partially funded by The Royal Thai Government.

\section{References}

1. Koper, R. and M. Specht: TenCompetence: Lifelong Competence Development and Learning. in:Competencies in Organizational E-Learning: Concepts and Tools, M.-A. Sicilia, Editor. (2007), Idea Group.

2. Shepard, L.A.: The Role of Assessment in a Learning Culture. Journal Information for Educational Researcher. Vol. 29, No. 7. (2000), 4-14.

3. Sitthisak, O., L. Gilbert, and H.C. Davis: Towards a competency model for adaptive assessment to support lifelong learning. in TENCompetence Workshop on Service Oriented Approaches and Lifelong Competence Development Infrastructures. (2007). Manchester, UK

4. Way, W.D.: Practical Questions in Introducing Computerized Adaptive Testing for K-12 Assessments, (2005)

5. Aroyo, L., P. Dolog, G.J. Houben, M. Kravcik, A.N.a.M. Nilsson, and F. Wild: Interoperability in Personalized Adaptive Learning. Education Technology and Society. Vol. 9, No. 2. (2006), 8-14.

6. Sitthisak, O., L. Gilbert, H.C. Davis, and M. Gobbi: Adapting health care competencies to a formal competency model. in the ICALT. (2007). Niigata, Japan: IEEE Computer Society Press.

7. UK Royal College of Nursing, Competencies. Available from http://www.rcn.org.uk/development/communities/specialisms/children_and_young_peopl e/resources/a-Z_of_resources/competencies

8. Ramritu, P.L. and A. Barnard: New nurse graduates' understanding of competence. International nursing review. Vol. No. (2001).

9. Defloor, T., A.V. Hecke, S. Verhaeghe, M. Gobert, E. Darras, and M. Grypdonck: The clinical nursing competences and their complexity in Belgian general hospitals. Journal of Advanced Nursing. Vol. 56, No. 6. (2006), 669-678.

10. Bloom, B.S. and D.R. Krathwohl: Taxonomy of educational objectives: The classification of educational goals by a committee of college and university examiners, ed. H.I.C. Domain. (1956), New York: Longman.

11. Merrill, M.D., Component Display Theory. Available from http://coe.sdsu.edu/eet/articles/cdt/index.htm

12. Krathwohl, D.R. and L. Anderson: A revision of bloom's taxonomy: An overview. Theory into Practice. Vol. 41, No. 4. (2002), 212-218.

13. Kunzmann, C.: Ontology-based Competence Management for Healthcare Training Planning: A Case Study. in Proceeding of the International Conference on Knowledge Management. (2006). Austria.

14. W3C, SKOS Core Guide. Available from http://www.w3.org/TR/swbp-skos-core-guide/

15. Johnson, B. and B. Shneiderman: Tree-Maps: a space-filling approach to the visualization of hierarchical information structures in Proceedings of the 2nd conference on Visualization '91 (1991). San Diego, California IEEE Computer Society Press.

16. McMillan, J.H.: Classroom Assessment: Principles and Practice for Effective Instruction. 4 ed. (2006): Pearson Technology Group. 
17. Rolfe, I. and J. MaPherson: Formative assessment: how am I doing? Lacent. Vol. 345, No. 8953. (1995), 837-839.

18. Sewell, J.: Diagnostic assessment within the Skills for Life strategy in 30th IAEA Conference (2004). Philadelphia

19. Brown, S., Using Formative Assessment to promote student learning. Available from http://www.ldu.leeds.ac.uk/news/events/documents/BrownPowerPoint.pdf

20. Nursing and Midwifery Council, Available from http://www.nmcuk.org/aFrameDisplay.aspx?DocumentID=171

21. Brusilovsky, P.: Adaptive Educational hypermedia, 8-12, (2001)

22. Falmagne, J.-C., E. Cosyn, J.-P. Doignon, and N. Thiery: The Assessment of Knowledge, in Theory and in Practice. in Integration of Knowledge Intensive Multi-Agent Systems. (2003).

23. Antoniou, G. and F.v. Harmelen: A Semantic Web Primer. (2004): The MIT Press.

24. IMS QTI, IMS Question and Test Interoperability Overview. Available from http://www.imsglobal.org/question/qtiv2p1pd2/imsqti_oviewv2p1pd2.html

25. Kalfoglou, Y.: Exploring Ontologies. Handbook of Software Engineering and Knowledge Engineering. Vol. 1: Fundamentals. (2001), Washington, D.C.: World Scientific Publishing.

26. Jovanovic, J., D. Gasevic, and V. Devedzic: Ontology-Based Automatic Annotation of Learning Content. Semantic Web and Information Systems. Vol. 2, No. 2. (2006), 91-119.

27. W3C, Resource Description Framework (RDF). Available from http://www.w3.org/RDF/

28. W3C, OWL Web Ontology Language (OWL). Available from http://www.w3.org/TR/owl-ref/ 